\title{
Propuesta técnica sobre el tratamiento del influente de la presa José Antonio Alzate a través un sistema lagunar aerobio
}

\section{Technical Proposal on the Treatment of the Influent of the José Antonio Alzate dam Using Aerobic Pond System}

\author{
S.G. Rosas de Alva \\ Departamento de Estudios de Posgrado. \\ Instituto Tecnológico de Toluca. Metepec, Estado de México. \\ E-mail:sergio.rosas01@cfe.gob.mx \\ I.D. Barceló-Quintal \\ Departamento de Ciencias Básicas. \\ Universidad Autónoma Metropolitana-Azcapotzalco. México. \\ E-mail:idbq@correo.azc.uam.mx \\ A.L. Bussy-Beaurain \\ Departamento de Ciencias Básicas. \\ Universidad Autónoma Metropolitana-Azcapotzalco. México. \\ E-mail:leemp@correo.azc.uam.mx \\ E. López-Galván \\ Departamento de Ciencias Básicas. \\ Universidad Autónoma Metropolitana-Azcapotzalco. México. \\ E-mail:loge@correo.azc.uam.mx
}

(Recibido: abril de 2008; aceptado: octubre de 2008)

\section{Resumen}

La descarga de aguas residuales a los sistemas acuáticos, sin previo tratamiento, es un problema generalizado en México; como es el caso del río Lerma en donde se sustituyó el aporte de agua limpia proveniente del sistema lagunar Almoloya-Lerma-San Bartolo, ubicado en el valle de Toluca Estado de México, por el agua residual del valle. Los intentos por limpiarla antes de su descarga han resultado infructuosos y esto ha causado que el río presente condiciones sépticas en todo su recorrido, incluyendo el primer cuerpo receptor que es la presa José Antonio Alzate (PJAA) creando condiciones peligrosas para la salud y limitando el uso de esta agua. En el presente trabajo se evalúa la eficiencia de tratamiento de un sistema lagunar aerobio experimental (SLE). Este sistema de tratamiento se construyó con 5 mamparas, las cuales formaron 6 canales internos. Ésta se operó en forma continua por espacio de 26 semanas, suministrándole en forma constante agua del Río Lerma. Se encontró que la eficiencia de reducción promedio, de la materia orgánica reportada como $\mathrm{DBO}_{5}$ fue de $83 \%$, removida en el primer canal después de 7 días de retención hidráulica. Esto comprobó que los canales no se comportan como lagunas en serie. Con los datos obtenidos, se aplicaron ecuaciones de diseño, obteniéndose el factor de decaimiento de la materia orgánica " $k$ " y el valor de dispersión " $d$ " específicos para el tipo de tratamiento y las condiciones ambientales del sitio. De esta forma, se calculó la superficie de la PJAA requerida para ser modificada en un sistema de tratamiento, basado en la eficiencia 
DOI: http://dx.doi.org/10.22201/fi.25940732e.2010.11n1.010

Propuesta técnica sobre el tratamiento del influente de la presa José Antonio Alzate a través un sistema lagunar aerobio

de reducción de $\mathrm{laDBO}_{5}$, obtenida experimentalmente. Así, se determinó que era necesaria una superficie de: 1281,6 hectáreas distribuidas en 13 lagunas sobre la superficie de la zona de entrada a la PJAA, este arreglo permitirá tratar un caudal de $8646.70 \mathrm{~m}^{3} / \mathrm{h}$. Esta superficie es mayor que la existente en la totalidad de la Presa, por lo que se evaluó el área disponible para hacer una propuesta real.

Descriptores: lagunas de estabilización aerobias, río Lerma, Presa José Antonio Alzate, demanda bioquímica de oxígeno, tiempo de retención hidráulico, número de dispersión.

\begin{abstract}
In México, discharge of waste water not treated is a common problem; this is the case of the Lerma River, where the original clean water contribution of the Almoloya-Lerma-San Bartolo timber-roof system, located in the valley of Toluca, State of México, was replaced by the wastewater of the valley. The attempts to clean the water before their spill have been unfruitful and this has caused the present anaerobic conditions of the river in its entire route including the first receiving stage that is the José Antonio Alzate Dam (PJAA), becoming a potential source of diseases and a useless water body. In this work we evaluate the treatment efficiency of experimental waste water pond system. The treatment system was constructed with 5 screens which formed 6 internal channels and it had an operation in continuous form by 26 weeks, providing it with continuously polluted water of the Lerma River. We found that the efficiency of reduction of the organic matter reported as $B O D_{5}$ was $83 \%$ and it is carried out in the first channel after 7 days of hydraulic retention verifying that the channels do not work like lagoons in series. With the collected data we calculated, through the application of design equations, the factor of decay of organic matter " $k$ " and the number of dispersion " $d$ ", specific for the type of treatment and environmental conditions of the site. The required surface of treatment calculated to reach the efficiency of reduction of the $B O D_{5}$ obtained experimentally. Thus, a surface of: 1281.6 hectares distributed in 13 lagoons that can deal with a volume 8 $646,70 \mathrm{~m}^{3} / \mathrm{h}$ was determined. Since this was a bigger surfaces than that of the dam, the available area was evaluated to make a proposal closer to reallity.
\end{abstract}

Keywords: Aerobic stabilization ponds, Lerma River, José Antonio Alzate dam, biochemical oxygen demand, hydraulic retention time, dispersion number.

\section{Introducción}

La Presa José Antonio Alzate ha sido ampliamente estudiada, a pesar de su alto grado de contaminación (Barceló, 2000), es empleada como reserva de agua para irrigación durante los meses de noviembre a abril y como depósito vacío para el control de inundaciones durante los meses de mayo a octubre. Maneja un caudal que fluctúa entre los $2 \mathrm{~m}^{3} / \mathrm{s}$ en época de estiaje y los $40 \mathrm{~m}^{3} / \mathrm{s}$ en época de lluvias. Desde el punto de vista de calidad del agua durante los meses de mayo y agosto, debido a los volúmenes del embalse, la dilución decrece significativamente y por lo tanto, las concentraciones de sólidos aumentan causando una mayor actividad de los procesos anaerobios (metanogénesis y formación de sulfuros) con el consecuente decaimiento de la calidad de este cuerpo de agua (Barceló, 2000). Es preciso aclarar que ocho de las catorce plantas de tratamiento construidas en el Estado de México están ubicadas en el área del lago y son exclusivamente Sistemas Lagunares de Estabilización (SLE). Estas se construyeron en los últimos 10 años para proteger la calidad del Río Lerma; sin embargo, ninguna de ellas funciona adecuadamente. Lo anterior fue diagnosticado y se concluyó que las fallas son de diseño, cortos circuitos hidráulicos, condiciones atmosféricas adversas y mala operación y mantenimiento (Lloyd et al., 2003).

El uso de lagunas de estabilización en países en desarrollo se ha incrementado notablemente; sin embargo, la tendencia a la construcción de plantas con tratamiento mecánico de alto costo sigue siendo una decisión tomada por los encargados de los sistemas municipales de 
DOI: http://dx.doi.org/10.22201/fi.25940732e.2010.11n1.010

S.G. Rosas de Alva, I.D. Barceló-Quintal, A.L. Bussy-Beaurain y E. López-Galván

saneamiento de agua en México. Lo anterior, se debe a que las experiencias previas con los sistemas lagunares no han sido satisfactorias a causa de su mal diseño y operación. Sin considerarse que el tratamiento de las aguas residuales con este tipo de sistemas consiste en una compleja simbiosis de algas, bacterias, protozoos e insectos (Beran y Kargi, 2005), además de que se han sobredimensionado las estructuras al emplear los modelos hidráulicos del tipo totalmente mezclado, así como el flujo de tipo pistón utilizados para el diseño y operación de estas estructuras (Sweeney et al., 2003). Con la finalidad de reducir estas incertidumbres se decidió construir un sistema lagunar aerobio a nivel piloto a un costado de la Presa José Antonio Alzate, y operarlo por 26 semanas con la finalidad de conocer con detalle su funcionamiento bajo condiciones climáticas reales para obtener los criterios y proponer la construcción de un sistema de tratamiento definitivo en dimensiones reales.

Los objetivos específicos del presente estudio fueron:

a) Construir y operar un sistema lagunar a nivel piloto bajo condiciones de campo.

b) Analizar el agua al entrar al sistema lagunar, durante su estancia y en la salida para identificar el comportamiento de la demanda bioquímica de oxígeno $\left(\mathrm{DBO}_{5}\right)$ y temperatura.

c) Obtener una constante cinética propia para las condiciones geográficas y climáticas de la zona de la PJAA.

d) Dimensionar un sistema lagunar para tratar el agua del río Lerma en su entrada a la presa José Antonio Alzate.

e) Evaluar la superficie de la PJAA y compararla con la superficie calculada.

\section{Material y método}

Área de estudio

La cuenca Río Lerma tiene su origen a partir de tres lagos poco profundos e interconectados, ubicados de norte a sur sobre el lado oriental del valle de Toluca, México. El lago más alto y el que se encuentra más al sur es conocido como Chignahuapan o Almoloya. Desde aquí el río desciende al norte hacia el lago Chimaliapan, también conocido como Lerma y luego sigue su ruta hacia el lago Chiconahuapan o San Bartolo en la parte noreste del valle. Juntos, estos lagos forman los inicios del sistema fluvial del Río Lerma, que corre hacia el oeste en dirección a la presa José Antonio Alzate (McClung y Sugiura, 2002). El sistema lagunar se desecó entre 1942 y 1951, debido a la construcción del acueducto y posterior bombeo del agua del Alto Lerma mexiquense hacia el Distrito Federal (Albores, 1995). En los primeros años de funcionamiento del Sistema Lerma, operando con gastos inferiores a los $5 \mathrm{~m}^{3} / \mathrm{s}$ la superficie ocupada por las lagunas disminuyó en tal forma que permitió la concesión agrícola de 2000 ha para el año 1957. Aunado a lo anterior, la problemática de salud del ecosistema de esta cuenca se inicia al realizar el abastecimiento de agua limpia a la población y a la industria del valle de Toluca, mediante la explotación de los mantos freáticos, ya que el $90 \%$ de ésta, es vertida al río Lerma en condiciones totalmente sépticas. Así los $4,3 \mathrm{~m}^{3} / \mathrm{s}$ de agua limpia que deberían ser aportados al Río Lerma por los escurrimientos de la laguna de Chignahuapan son sustituidos con agua residual que inicia su vertido en el nacimiento original del río, por las descargas del pueblo de Almoloya del Río.

La Presa José Antonio Alzate, se encuentra en el tramo comprendido entre la carretera Toluca-Naucalpan en los municipios de Ixtlahuaca y Almoloya de Juárez, aproximadamente a $25 \mathrm{~km}$ al norte de Toluca, sobre el Río Lerma y fue construida en el periodo de 1960 a 1962 (figura 1). Este cuerpo de agua se forma al embalsar el río Lerma por medio de una cortina que cuenta con 19 metros de altura. La capacidad total del embalse es de 36,3 millones de metros cúbicos que incluye 8 millones de metros cúbicos para el depósito de azolve. La superficie total del lago formado por la presa es de 1050 ha (Barceló, 2000; Ávila, 2001).

\section{Diseño experimental}

\section{Construcción del sistema lagunar piloto}

Las pruebas se realizaron en una laguna de estabilización experimental construida en el costado sur sureste de la presa José Antonio Alzate en las coordenadas $19^{\circ} 27^{\prime \prime} 44^{\prime}$ de latitud norte y $99^{\circ} 39^{\prime} 03^{\prime \prime}$ de longitud oeste y en una altitud de $2650 \mathrm{msnm}$. La instalación consistió de la excavación, nivelación y aplanado de una fosa, cuyo interior fue impermeabilizado con cemento tanto en el fondo como en las paredes. Se construyeron cinco mamparas, lo que dividió a la laguna en 6 canales (figura 4). Las características dimensionales aparecen en el cuadro 1. Como sistema de suministro con el agua del río Lerma se utilizó un contenedor de plástico de $1,2 \mathrm{~m}^{3}$, el cual se instaló en un desnivel construido con tabique-cemento a una altura de $0,52 \mathrm{~m}$, los gastos del agua 
cruda se regularon con una válvula de compuerta. La salida del agua tratada se condujo nuevamente a la PJAA por medio de un canal de $5 \mathrm{~m}$ de longitud, $0,50 \mathrm{~m}$ de ancho con una profundidad máxima en su límite con el sistema lagunar de 1,65 m y una profundidad mínima de $0,15 \mathrm{~m}$ en su llegada al sistema.

\section{Operación del sistema lagunar experimental}

Se establecieron 7 puntos de muestreo en la entrada y salida del sistema, así como en cada uno de los canales formados por las mamparas (figura 2). Se operó con una profundidad que fluctuó entre 1,03 m y 1,09 m y se reguló un gasto de $0,432 \mathrm{~m}^{3} /$ día.

La salida del agua tratada se llevó a cabo por medio de un sistema de válvulas de compuerta instaladas en la parte final del canal número 7.

Después de 14 días de operación, se observó un cambio en las condiciones físicas y biológicas del agua por la aparición de algas verdes, y con ello, la presencia de oxígeno disuelto en todo el cuerpo de agua, cambiando las condiciones anaerobias del sistema a condiciones totalmente aerobias (figura 3 ).

\section{Métodos de muestreo y análisis}

Se tomaron muestras en el sistema lagunar durante 26 semanas en 7 puntos diferentes del sistema lagunar. Se analizó la demanda bioquímica de oxígeno $\left(\mathrm{DBO}_{5}\right)$, se midió el oxígeno disuelto y la temperatura del agua, que fueron indispensables para establecer la cinética de degradación.

La $\mathrm{DBO}_{5}$ se analizó de acuerdo con el método establecido en la norma mexicana NMX-AA_028-SCI-2001. El oxigeno se midió mediante un analizador de oxígeno disuelto con termopar integrado, marca YSI-52 y el sensor de temperatura se calibró con un termómetro de vidrio.

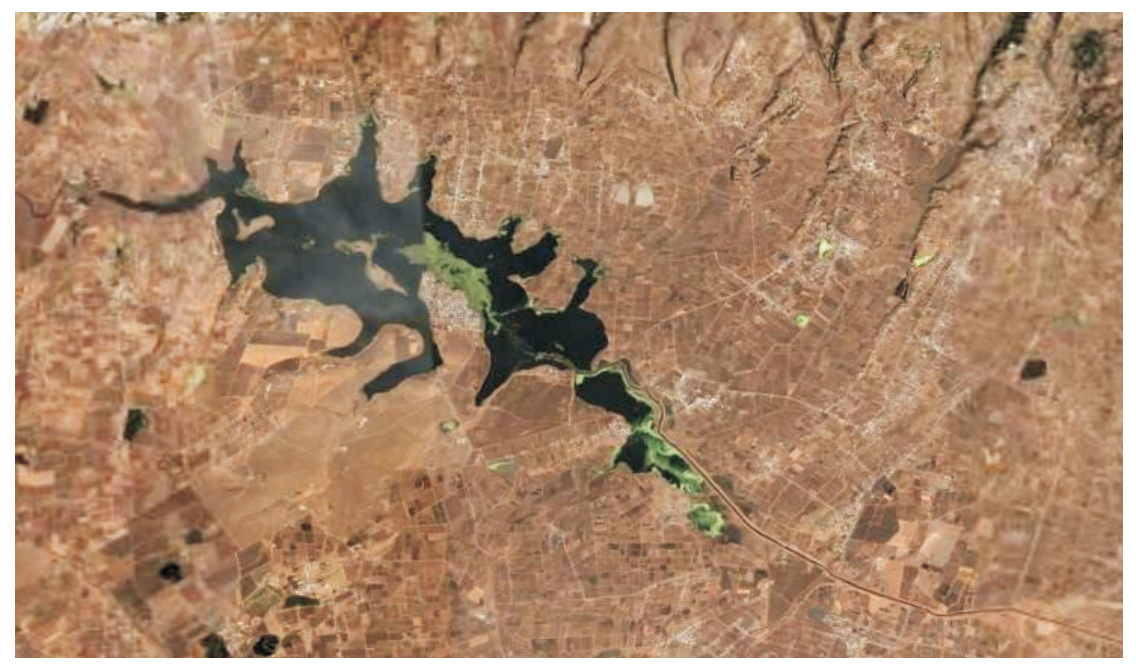

Figura 1. Presa José Antonio Alzate

Cuadro 1. Características generales del sistema lagunar experimental

\begin{tabular}{ccccc}
\hline Canales & Longitud $\mathrm{m}$ & Ancho $\mathrm{m}$ & Profundidad $\mathrm{m}$ & Volumen $\mathrm{m}^{3}$ \\
\hline 1 & 4,40 & 0,70 & 1,03 & 3,1724 \\
2 & 5,40 & 0,54 & 1,08 & 3,1492 \\
3 & 5,40 & 0,57 & 1,08 & 3,3242 \\
4 & 5,40 & 0,59 & 1,07 & 3,4090 \\
5 & 5,40 & 0,60 & 1,09 & 3,5316 \\
6 & 5,40 & 0,72 & 1,07 & 4,1601 \\
\hline
\end{tabular}




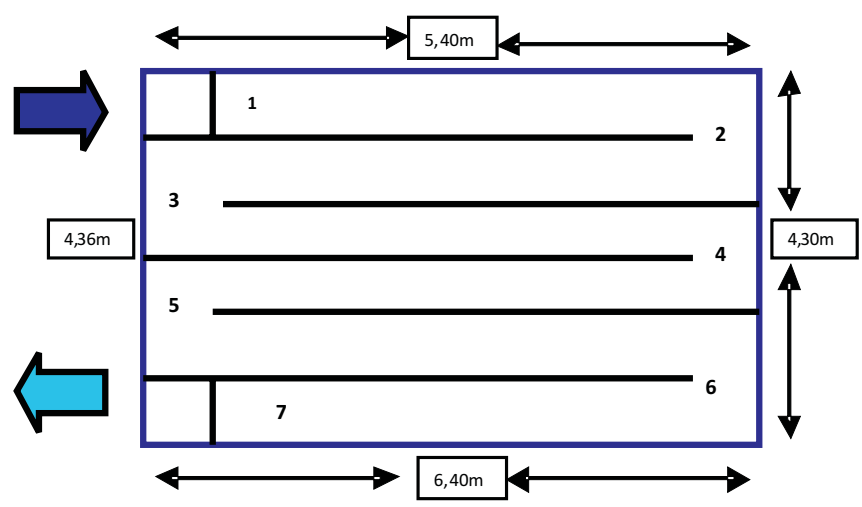

Figura 2. Vista de planta del sistema lagunar experimental

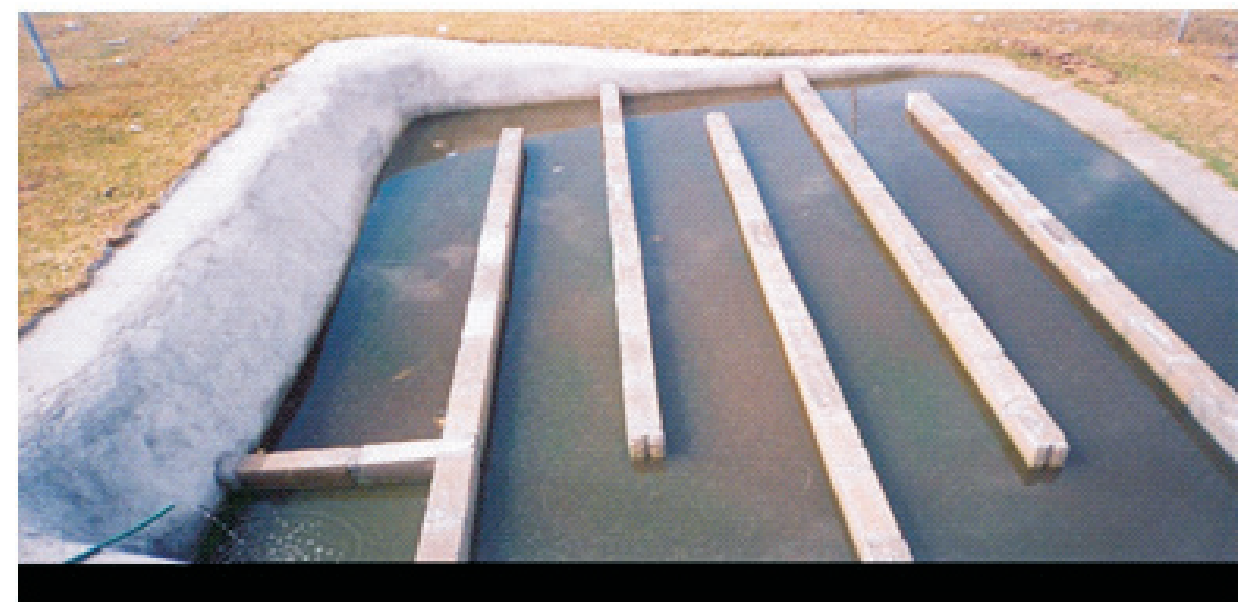

Figura 3. Crecimiento de algas en el sistema lagunar II

\section{Demanda bioquímica de oxígeno}

Su reducción en el sistema lagunar se estabilizó después de 7 días y 13 horas de retención en el primer canal. Posteriormente, se mantuvo sin variaciones significativas en los demás canales (figura 4).

Los valores de la $\mathrm{DBO}_{5}$ en la entrada al sistema, encontraron entre 60 y $200 \mathrm{mg} / \mathrm{L}$. Su remoción fue de $82 \%$. Las concentraciones después del canal 1 no variaron conforme la calidad del agua de alimentación al sistema lagunar.

Al diluirse el agua de alimentación con agua de lluvia en el intervalo de muestras 21 al 26, la $\mathrm{DBO}_{5}$ de entrada disminuyó a concentraciones muy cercanas a la $\mathrm{DBO}_{5}$ de la salida, debido a la presencia de lluvias. No obstante, se puede observar poca variación en la calidad del agua del efluente, esto significa que el ecosistema lagunar es muy estable y se adecua a la fluctuación en la concentración de la materia orgánica disuelta en su entrada.

\section{Temperatura}

La temperatura junto con el oxígeno disuelto son dos factores determinantes en el comportamiento de la cinética de degradación de la materia orgánica por medio de microorganismos (Fritz et al., 1980).

La temperatura diaria en los 3 niveles de profundidad (fondo, medio y superficie) en los 7 puntos de dirección, se promediaron por semana y graficaron. 


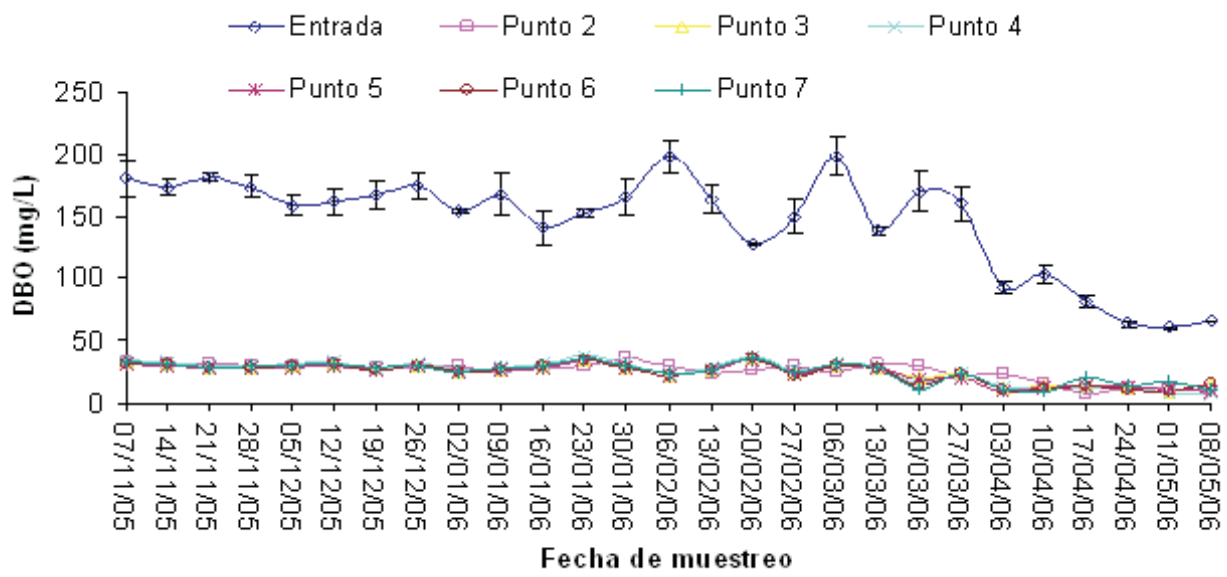

Figura 4. Comportamiento de la $\mathrm{DBO}_{5}$ en el sistema lagunar

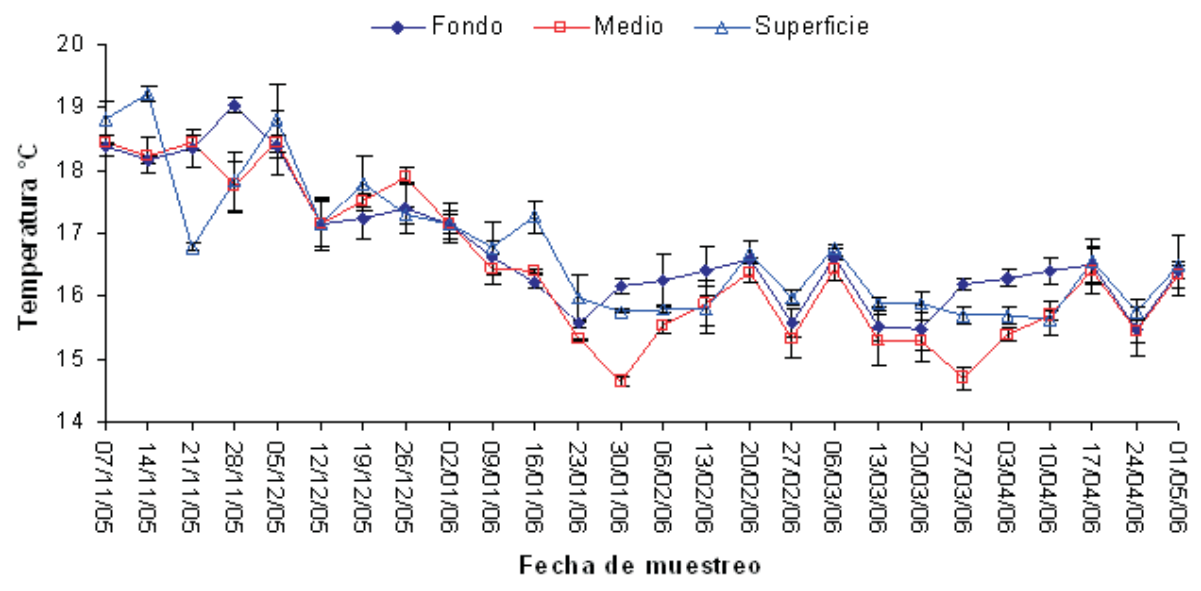

Figura 5. Comportamiento de la temperatura

El comportamiento de la temperatura en el sistema lagunar experimental es característico de los cuerpos de agua estáticos, debido a la baja velocidad de su desplazamiento (figura 5). Es decir, la columna de agua presenta una estratificación diurna ligera dentro de los periodos de insolación y los periodos nocturnos de disminución de la temperatura atmosférica $\left(9^{\circ} \mathrm{C}\right)$, en general la columna de agua permanece a la misma temperatura en la cual el sistema mesofílico microbiano puede trabajar (14,7 a 19,3ㅇ) (Armas, 2002).

\section{Oxígeno disuelto}

El tratamiento efectuado en las lagunas facultativas es el resultado de una compleja interrelación de diferentes especies de bacterias y algas que existen en la laguna y que tienen un patrón ecológico diferente a los cultivos en laboratorio, en el cual el requerimiento de oxígeno es cubierto con el oxígeno fotosintético. Obviamente, en estos sistemas naturales la velocidad de crecimiento y otras actividades microbianas son afectadas por las variaciones diurnas de intensidad de luz solar, oxígeno disuelto y temperatura (Kayombo et al., 2003). Lo anterior, se confirma con la presencia de oxígeno en todos los niveles del sistema experimental, como puede observarse en la figura 6. Las concentraciones de oxígeno disuelto en los tres niveles del cuerpo de agua y en los 7 puntos geométricos de la laguna, arrojaron datos que fluctúan entre $1,5 \mathrm{mg} / \mathrm{L}$ y $3,2 \mathrm{mg} / \mathrm{L}$ lo que demuestra que el sistema de tratamiento aerobio es sostenible en las condiciones en las que se encuentra el agua del río (figura 6). 


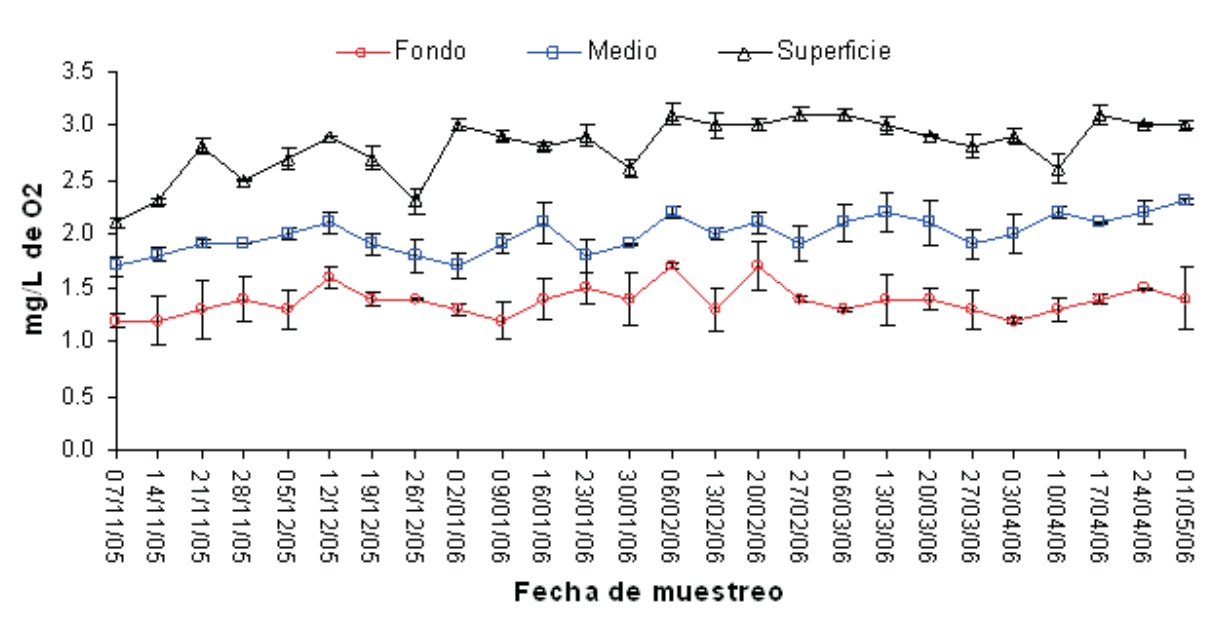

Figura 6. Comportamiento del oxígeno disuelto

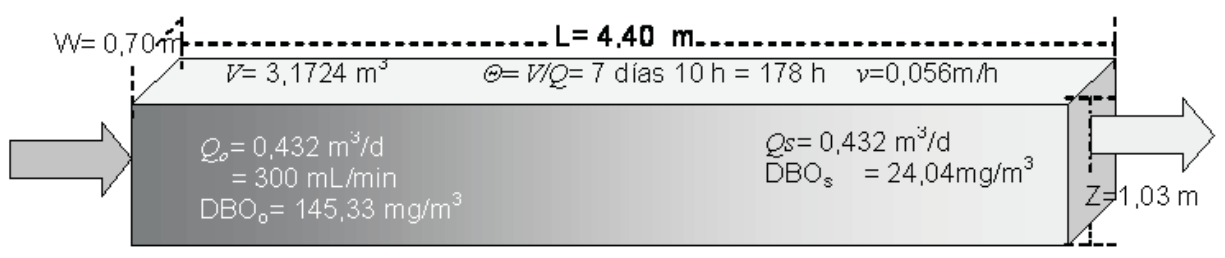

Figura 7. Características del canal 1 del sistema lagunar

\section{Cálculos y dimensionamiento del sistema}

Una de las principales restricciones para construir sistemas lagunares de estabilización es la superficie disponible sobre la cual realizar su construcción (Marais et al., 1961; Gloyna, 1973; Romero, 1994; Saenz, 2001). Por otra parte, trabajos de investigación han demostrado que la PJAA tiene la capacidad propia para llevar a cabo diferentes fenómenos de transporte que auxilian en la depuración del agua del río Lerma durante su almacenamiento y posterior liberación. (Barceló, 2000). Estos dos factores dirigen la atención a proponer una modificación física de la PJAA con el propósito de cambiar su comportamiento hidráulico de acuerdo con los datos experimentales obtenidos en el presente trabajo y siguiendo el método de diseño de lagunas de acuerdo con los criterios de diferentes investigadores. Es importante destacar que los análisis de los comportamientos de los sistemas lagunares experimentales arrojaron que la estabilización de la materia orgánica se llevó a cabo en el primer canal formado por la división con mamparas, por lo que el análisis de diseño se llevó a cabo sobre lo ocurrido en este canal. Como se puede observar, en la figura 4 en el sistema lagunar el comportamiento de la materia orgánica representada por la $\mathrm{DBO}_{5}$ experimenta una reducción en el punto de muestreo 2 , manteniéndose casi constante en los siguientes puntos. Por este motivo se analizó este canal para el diseño final del sistema lagunar. El resumen de las características hidráulicas del canal se representa en la figura 7.

\section{Cálculo del número de dispersión}

Para determinar el tipo de flujo que se presentó durante la fase experimental, se calculó el factor de dispersión $d$ por medio de las ecuaciones 1 a 4 del cuadro 2 .

Las cuatro demuestran que el flujo es disperso, es decir, no se puede considerar como flujo totalmente mezclado ni como flujo pistón, ya que la $d$ obtenida, tiene un valor muy cercano a cero. Se seleccionaron en la literatura ecuaciones de remoción obtenidas bajo este régimen de flujo.

Además se utilizaron los promedios de temperaturas del agua en el sistema lagunar. Se eligieron las ecuaciones 5 a la 8 del cuadro 3 para flujo disperso, en esta tabla se indican los valores de la constante de remoción para los modelos de Yáñez (1993), Sánchez (1992), Sáenz (2001) y el obtenido en el presente experimento. Con estos datos se calculó el tiempo de retención hidráulico. 
DOI: http://dx.doi.org/10.22201/fi.25940732e.2010.11n1.010

Propuesta técnica sobre el tratamiento del influente de la presa José Antonio Alzate a través un sistema lagunar aerobio

Cuadro 2. Números de dispersión de acuerdo con diferentes investigadores

\begin{tabular}{cccc}
\hline Ecuación & Referencia & Resultado \\
\hline$d=\frac{1}{(L / W)}$ & 2.5 & $\begin{array}{c}\text { Von Sperling } \\
(2000)\end{array}$ & $\mathrm{d}=0,159$ \\
$d=\frac{L / W}{0,261+0,254(L / W)+1,014(L / W)^{2}}$ & 2.6 & $\begin{array}{c}\text { Yañez } \\
(1993)\end{array}$ & $\mathrm{d}=0,149$ \\
$d=0,102\left[\frac{3(W+2 Z) t v}{4 L W Z}\right]^{-0,410}\left[\frac{Z}{L}\right]\left[\frac{Z}{W}\right]^{\left(0,981+1,855 \frac{Z}{W}\right)}$ & 2.7 & $\begin{array}{c}\text { Agunwamba } \\
(1992)\end{array}$ & $\mathrm{d}=0,0031$ \\
$d=\frac{0,184 t v(W+2 Z)^{-0,410} W^{1,511}}{(L Z)^{1,489}}$ & & $\begin{array}{c}\text { Polprasert y Bahattarai } \\
(1985)\end{array}$ & $\mathrm{d}=0,033$ \\
\hline
\end{tabular}

Cuadro 3. Ecuaciones para obtener constantes de remoción

\begin{tabular}{cccc}
\hline Ecuación & Núm. & Referencia & Kb $\left(d^{-1}\right)$ \\
\hline$K_{b}=0,84(1,07)^{t-20}$ & 5 & Yáñez (1993) & 0.685 \\
$K_{b}=1,1(1,07)^{t-20}$ & 6 & Sánchez (1992) & 0.897 \\
$K_{b}=0,623(1,037)^{t-20}$ & 7 & Sáenz (2001) & 0.558 \\
$K_{b}=0,8(1,07)^{t-20}$ & 8 & Experimental & 0.632 \\
\hline
\end{tabular}

\section{Cálculo del tiempo de retención hidráulico}

Con la finalidad de seleccionar la ecuación adecuada para el dimensionamiento del sistema lagunar en la PJAA, se probaron los resultados obtenidos con las tres ecuaciones de remoción y se utilizaron los datos experimentales de la $\mathrm{DBO}_{5}$ en la entrada y en la salida del sistema lagunar en condiciones de mayor carga orgánica en la entrada y mayor eficiencia de remoción, se aplicaron a la ecuación de Marais (1970) con la finalidad de obtener el tiempo de retención teórico y compararlo con el tiempo de retención real del canal 1 en el sistema lagunar piloto, asumiendo que el tiempo de retención calculado más cercano al tiempo de retención experimental, dará la constante que se utilizará en el dimensionamiento real (ecuación 9).

$$
t=\frac{1}{K}\left[\left(\frac{S_{0}}{S_{e}}\right)-1\right] .
$$

En el cuadro 4 se resume la utilización de las diferentes constantes de remoción en la ecuación 9.

La constante de remoción obtenida con el modelo de Yáñez en el que se obtuvo un tiempo de retención de 7 días con 8 horas, fue la que mostró la mayor aproximación con el tiempo de retención obtenido experimentalmente en el primer canal que fue de 7 días con 13 horas.

La superficie en hectáreas que se requiere para instalar un sistema lagunar con un tiempo de retención de 7 días y 10 horas y un caudal a tratar de $20 \mathrm{~m}^{3} / \mathrm{s}$ se obtuvo sustituyendo y desarrollando en la ecuación 10.

$$
\begin{aligned}
& A=\frac{t Q_{O}}{h} \\
& A=\frac{(178 h)\left(72000 \mathrm{~m}^{3} / h\right)}{1 \mathrm{~m}}=12816000 \mathrm{~m}^{2} .
\end{aligned}
$$

Resultando una superficie requerida de: 1 281,6 hectáreas.

\section{Consideración del área disponible en la presa Alzate para la construcción del sistema lagunar}

La superficie total de la PJAA es de 1050 hectáreas (CNA, 1993) y ha sido dividida para fines de estudio en 6 zonas (Barceló, 2000). La zona en donde el río Lerma se conecta a la PJAA es conocida como zona A y presenta condiciones de profundidad óptimas para desarrollar trabajos de nivelación y formación de diques para la 
DOI: http://dx.doi.org/10.22201/fi.25940732e.2010.11n1.010

S.G. Rosas de Alva, I.D. Barceló-Quintal, A.L. Bussy-Beaurain y E. López-Galván

Cuadro 4. Ecuaciones para obtener tiempos de retención

\begin{tabular}{cccc}
\hline Ecuación & Núm. & Referencia & t días \\
\hline$t=\frac{1}{0,685 d^{-1}}\left[\left(\frac{145 m g / L}{24 m g / L}\right)-1\right]$ & 9 & Yáñez (1992) & 7,36 \\
$t=\frac{1}{0,897 d^{-1}}\left[\left(\frac{145 m g / L}{24 m g / L}\right)-1\right]$ & 10 & Sánchez (1992) & 5,62 \\
$t=\frac{1}{0,558 d^{-1}}\left[\left(\frac{145 m g / L}{24 m g / L}\right)-1\right]$ & 11 & Sáenz (2001) & 9,04 \\
\hline
\end{tabular}

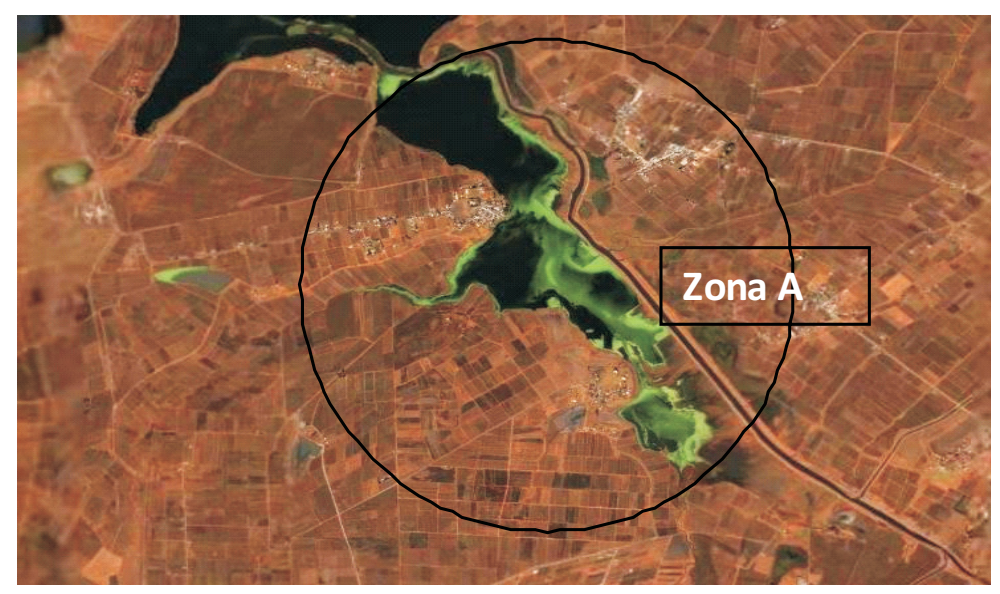

Figura 8. Zona A de la Presa José Antonio Alzate

corrección hidráulica, tiene una superficie de 474,06 hectáreas (figura 8) y una profundidad media de 1,5 m.

\section{Sistema lagunar de estabilización}

Considerando el comportamiento en el sistema lagunar experimental, se calcularon lagunas con una profundidad de $1 \mathrm{~m}$ y los tiempos de retención hidráulicos experimentales de 7,61 días (figura 9). Utilizando la ecuación 12 se obtuvieron los gastos que pueden ser tratados en el área disponible resultando un sistema de 13 lagunas distribuidas en el polígono resultante del análisis de la imagen de satélite de la zona A. De acuerdo con el área disponible en el polígono se obtienen 3 paquetes lagunares con diferentes capacidades de tratamiento como se muestra en el cuadro 5.
La capacidad de tratamiento de cada paquete lagunar aparece en el cuadro 6.

La capacidad total de tratamiento mediante lagunas aerobias, que es posible instalar en la zona de entrada de la Presa Antonio Alzate, es de $8646.70 \mathrm{~m}^{3} / \mathrm{h}(2.399$ $\mathrm{m}^{3} / \mathrm{s}$ ), mientras que el gasto medio en época de estiaje es de $2 \mathrm{~m}^{3} / \mathrm{s}\left(2 \mathrm{~m}^{3} / \mathrm{s}\right)$ y en época de lluvias de $20 \mathrm{~m}^{3} / \mathrm{s}$.

Se determinó que es posible tratar el 100\% del agua que entra a la PJAA en época de estiaje, hasta el 11.72\% en época de lluvias. Lo anterior, aunque no es una solución definitiva al problema de la cuenca, si una ayuda con gran potencial de recuperación del ecosistema de este vaso, ya que, como se ha demostrado en los trabajos previos (Barceló, 2000; Ávila, 2001) la hidráulica de la presa permite que los fenómenos de transporte tiendan a estabilizar este cuerpo de agua. 


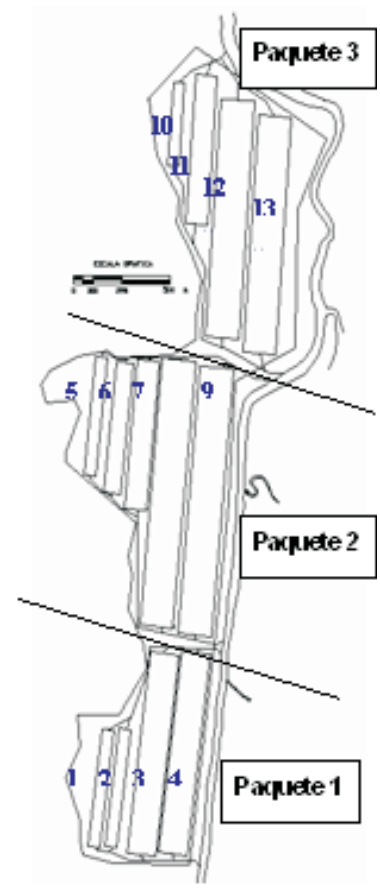

Figura 9. Distribución geométrica de los paquetes lagunares en la zona A

Cuadro 5. Dimensiones del sistema lagunar de estabilización en el área disponible

\begin{tabular}{|c|c|c|c|c|c|}
\hline Lagunas & $\begin{array}{l}\text { Largo } \\
(\mathrm{m})\end{array}$ & $\begin{array}{c}\text { Ancho } \\
(\mathrm{m})\end{array}$ & $\begin{array}{l}\text { Profundidad } \\
(\mathrm{m})\end{array}$ & Núm. de Lagunas & $\begin{array}{c}\text { Capacidad de } \\
\text { tratamiento }\left(\mathrm{m}^{3} / \mathrm{h}\right)\end{array}$ \\
\hline $1,2,5$ & 571.43 & 59.42 & 1 & 3 & 601.95 \\
\hline 3,4 & 971.43 & 148.57 & 1 & 2 & 1705.77 \\
\hline 6 & 628.57 & 85.71 & 1 & 1 & 318.37 \\
\hline 7,11 & 714.30 & 114.30 & 1 & 2 & 964.95 \\
\hline 8,9 & 1285.71 & 171.43 & 1 & 2 & 2605.01 \\
\hline 10 & 400.00 & 57.14 & 1 & 1 & 135.06 \\
\hline 12,13 & 1142.87 & 171.43 & 1 & 2 & 2315.60 \\
\hline
\end{tabular}

Cuadro 6. Capacidad de tratamiento de cada paquete lagunar

\begin{tabular}{ccc}
\hline \multirow{2}{*}{ Paquete lagunar } & \multicolumn{2}{c}{ Capacidad de tratamiento } \\
& $\mathrm{m}^{3} / \mathrm{h}$ & $\mathrm{m}^{3} / \mathrm{s}$ \\
\hline 1 & 2107.06 & 0.585 \\
2 & 3606.52 & 1.000 \\
3 & 2933.12 & 0.814 \\
\hline
\end{tabular}


DOI: http://dx.doi.org/10.22201/fi.25940732e.2010.11n1.010

S.G. Rosas de Alva, I.D. Barceló-Quintal, A.L. Bussy-Beaurain y E. López-Galván

\section{Conclusiones}

El río Lerma dejó de existir como tal desde la década de 1940, cuando se inició el envío de $4,5 \mathrm{~m}^{3}$ /s del acuífero del alto Lerma a la Ciudad de México, abatiéndose el manto freático e iniciando la descarga de agua residual municipal sin tratar al lecho del río, convirtiéndose en un canal conductor de aguas residuales que desemboca en la presa José Antonio Alzate como primer cuerpo receptor.

Los intentos de sanear este importante cuerpo de agua han sido infructuosos, ya que se ha considerado que el volumen de agua a tratar es extremadamente alto, lo cual conlleva a la inversión de grandes cantidades de dinero para el caso de tratamientos mecánicos convencionales.

La incertidumbre sobre la eficiencia de los sistemas lagunares en agua altamente contaminada y bajo las condiciones geográficas y climáticas de la zona del Valle de Toluca es eliminada al realizar experimentos de tratamiento en el sitio, ya que el sistema experimental instalado, presentó un rápido cambio de condiciones anaerobias a condiciones aerobias con la aparición de algas y la presencia de oxígeno en la totalidad del cuerpo de agua. La eliminación del $83 \%$ de la $\mathrm{DBO}_{5}$ presente, dio los datos necesarios para el cálculo de un sistema lagunar formado por 3 paquetes con un total de 13 lagunas con diferentes dimensiones que guardan las proporciones L/W menores que 7 de acuerdo con el sistema experimental y distribuidas en la zona de entrada de la presa José Antonio Alzate. Estos paquetes lagunares tienen la capacidad de tratar el 11,7\% del caudal medio de río Lerma en la época de lluvias y en un 100\% en la época de estiaje. Con el comportamiento del sistema lagunar bajo condiciones de temperatura reales, se obtuvieron los datos necesarios para obtener una constante de degradación que puede utilizarse en el futuro para el cálculo de sistemas lagunares exclusivamente en la zona del Valle de Toluca. También se desarrolló una herramienta predictiva para el comportamiento de la $\mathrm{DBO}_{5}$ que podría utilizarse en futuros proyectos. La desaparición del ecosistema en la cuenca alta del Lerma tiene su origen desde el sistema lagunar que originalmente lo alimentaba, por lo que se recomienda realizar una investigación de los aportes de agua servida al lecho del Lerma con el fin de conocer las características de tratabilidad, así como trabajos experimentales con sistemas de tratamiento a escala en los sitios de interés. Es importante considerar que se establezcan aguas arriba sistemas lagunares semejantes al propuesto, de manera que el agua llegaría a la PJAA con mejor calidad, optimizando el sistema de tratamiento. Por otro lado, es importante que se haga cumplir la Ley de Aguas Nacionales a las autoridades municipales del Valle de Toluca con el fin de reducir la carga biológica en toda la cuenca del Alto Lerma; para ello, se recomienda que se otorgue prioridad a los sistemas de tratamiento natural hasta donde lo permita la situación técnica y que se aumente el presupuesto a la Comisión de Agua Potable y Alcantarillado del Estado de México con la finalidad de operar y mantener los sistemas de tratamiento que se instalarán en el futuro. Por último, con la finalidad de obtener financiamiento para la corrección hidráulica de la zona "A" de la PJAA, se recomienda hacer un estudio de la emisión de metano, debido a las condiciones anaerobias actuales de este cuerpo de agua y la cantidad que dejaría de producirse en el caso de cambiar a condiciones aerobias para presentarlo en el Comité de Cambio Climático de México y solicitar financiamiento por medio del mercado de bonos de bióxido de carbono y del metano.

\section{Agradecimientos}

Al Consejo Nacional de Ciencia y Tecnología por el otorgamiento del soporte financiero del proyecto. Nuestro agradecimiento a la Universidad Autónoma Metropolitana unidad Azcapozalco por su apoyo con los recursos físicos y de logística. Le agradecemos al Instituto Tecnológico de Toluca su apoyo en el desarrollo del proyecto.

\section{Referencias}

Agunwamba J.C., Egbuniwe N., Ademilyui J.O. Prediction of the Dispersion Number in Waste Stabilization Ponds. Water Research, London, 26(1):85-89. Enero 1992.

Albores-Zárate B.A. Tules y sirenas. El impacto ecológico y cultural de la industrialización en el Alto Lerma, México. El Colegio Mexiquense, A.C. Gobierno del Estado de México. Secretaría de Ecología. Estado de México. 1995.

Avila P. Dinámica de metales pesados no esenciales en la interacción agua-sedimento-biota de la presa José Antonio Alzate. Tesis (Doctorado). Facultad de Ingeniería, Universidad Autónoma del Estado de México. 2001.

Barceló I. Estudio de la movilidad de Ca, Cd, Cu, Fe, Mn, Pb y Zn en sedimentos de la presa José Antonio Alzate en el Estado de México. Tesis (Doctorado). Facultad de Ingeniería, Universidad Autónoma del Estado de México. 2000.

Beran B., Kargi F. A Dynamic Model for Wastewater Stabilization Ponds. Ecological. Modelling, 174:225-240. 2002. 
CNA, Comisión Nacional del Agua. Actualización de la información de los acuíferos del alto Lerma (valles de Toluca e Ixtlahuaca, Edo. de México) y la adaptación de su modelo DAS al lenguaje basic. Documento de la DGCOH. México. S.n.p., 1993.

Fritz J.J., Middleton A.C., Meredith D.C. Dynamic Process Modeling of Waste Water Stabilization Ponds, Water Research, 14(5). 1980.

Gloyna E.F. Estanques de estabilización de aguas residuales. OMS. Ginebra, Suiza. 1973.

Kayombo S., Mbwette T.S.A., Katima J.H.Y., Jórgensen S.E. Effects of Substrate Concentrations on the Growth of Heterotrophic Bacteria and Algae in Secondary Facultative Ponds. Water Research 37:2937-2943.1973.

Lloyd B.J., Letner A.R., Vorkas C.A., Guganesharajah R.K. Under-Perfomance Evaluation and Rehabilitation Strategy for Waste Stabilization Ponds in Mexico. Water Science and Technology. 48(2):35-43. 2003.

Marais G.V.R., Shaw V.A. A Rational Theory for the Design of Sewage Stabilization Ponds in Central and South Africa. Trans. S. Africa Inst. Civil. Engrs. 13:11. 1961.

Marais G.V.R. Dynamic Behavior of Oxidation Ponds. Proc. $2^{\text {nd }}$ Int. Symposium on Waste Treatment Lagoons. Kansas City, Kan. 1970.

Mcclung de Tapia E., Sugiura Y. Prehispanic Life in a Man-made Island Habitat in Chignahuapan Marsh, Santa Cruz Atizapán, State of México, México. Informe de beca FAMSI. 2002.
Polprasert Ch., Bahattarai K. Dispersion Model for Waste Stabilization Ponds. Journal of Environmental Engineering, ASCE, 3(1). 1985.

Romero J.A. Acuitratamiento por lagunas de estabilización. Editorial Escuela Colombiana de Ingeniería, Colombia. Capítulo 2. 1994.

Saenz R.F. Elongated Stabilization Ponds with Partitions: A Solution for Small Communities Sewage Disponsal. POND0105.wpd., 2001.

Sánchez A., Maturana J. Evaluación de las lagunas de estabilización, Sistema Esmeralda. En: memorias técnicas, XXIII Congreso Interamericano de Ingeniería Sanitaria y Ambiental. Vol. II parte 2., 1992.

Sweeney D.G., Cromar N.J., Nixon J.B., Ta C.T., Fallowfield H.J. The Spatial Significance of Water Quality Indicators in Waste Stabilization Ponds-Limitations of Residence Time Distribution Analysis in Predicting Treatment Efficiency. Wat. Sci. Tech. 48(2):211-218. 2003.

Von Sperling M. Propuesta de modelos para estimar la remoción de coliformes en lagunas de estabilización, con base en datos de 33 lagunas brasileñas. Revista Engenharia Sanitaria e Ambiental, ABES. 2000.

Yañez F. Lagunas de estabilización. Teoría diseño, evaluación y mantenimiento. Empresa Pública Municipal de Teléfonos, Agua Potable y Alcantarillado de Cuenca Ecuador, ETAPA.1993. 
DOI: http://dx.doi.org/10.22201/fi.25940732e.2010.11n1.010

S.G. Rosas de Alva, I.D. Barceló-Quintal, A.L. Bussy-Beaurain y E. López-Galván

\section{Semblanza de los autores}

Sergio Gerardo Rosas de Alva. Obtuvo el título de químico (1984) y el grado de maestro en ciencias con especialidad en ingeniería ambiental (2003) en la Universidad Autónoma de Nuevo León. Asimismo, el grado de doctor en ciencias con especialidad en ingeniería ambiental en el Instituto Tecnológico de Toluca (2009). Actualmente es el jefe del Departamento de Control Ambiental de la Subdirección de Generación de la Comisión Federal de Electricidad. Sus principales áreas de trabajo son la recuperación de agua y la interacción de las actividades de generación de energía eléctrica con los ecosistemas vecinos.

Icela Dagmar Barceló-Quintal. Ingeniera química egresada de la Escuela de Química de la Universidad de Yucatán en 1972. Maestra en ciencias con especialidad en química inorgánica, egresada de la Facultad de Química de la Universidad de Guanajuato en 1975. Doctora en ingeniería en ciencias del agua en el Centro Interamericano de Recursos del Agua (CIRA) de la Facultad de Ingeniería de la Universidad Autónoma del Estado de México en el año 2000, jefa del Área de Química y Fisicoquímica Ambiental, UAM-Azcapotzalco. Profesora investigador titular de la UAM-Azcapotzalco. Ha presentado ponencias en foros nacionales e Internacionales. Ha dirigido tesis de licenciatura, maestría y doctorado. Ha publicado en memorias de congresos nacionales e internacionales, coautora de artículos en revistas especializadas nacionales e internacionales.

Anne-Laure Bussy-Beaurain. Doctora en ciencias y técnicas del medio ambiente, profesora investigadora de la UAM-Azcapotzalco. Ha presentado ponencias nacionales e internacionales. Ha dirigido tesis de licenciatura. Ha publicado en memorias de congresos nacionales e internacionales, coautora de artículos en revistas especializadas nacionales.

Edgar López-Galván. Ingeniero químico, maestro en ingeniería, profesor investigador de la UAM-A, estudiante de doctorado en ingeniería ambiental. Ha dirigido tesis a nivel licenciatura y ha presentado ponencias en congresos nacionales e internacionales. 